\title{
Effect of Protrusions on Cathodic-Arc-Attachment Mode in High-Pressure Arc Discharges
}

\author{
Mikhail S. Benilov, Senior Member, IEEE, Mário D. Cunha, and Maria J. Faria
}

\begin{abstract}
It is shown via computer simulations that a protrusion on a thermionic arc cathode may dramatically change the pattern of steady-state modes of current transfer. This happens through a bifurcation of a special type occurring at certain geometry of the cathode and may play a role in operation of electrodes of compact high-intensity discharge lamps.
\end{abstract}

Index Terms-Arc attachment, arc cathode, bifurcation of current transfer, bifurcation theory, high-pressure arc.

$\mathbf{C}$ ONSIDERABLE advances in theoretical and experimental investigation of the plasma-cathode interaction in high-pressure arc discharges have been achieved during the last decade [1]. Simulation methods, available at present and validated experimentally, allow one to reliably describe many aspects of operation of thermionic cathodes.

A challenging task is to simulate operation of cathodes of complex geometries, which are of practical interest, in particular, those with protrusions [2], [3]. Results of such simulations are reported in this paper.

Steady-state modes of current transfer have been calculated with the use of the commercial software COMSOL Multiphysics, as described in [4]. Stability of each of the steady-state modes has been investigated, as described in [5].

In Fig. 1, current-voltage characteristics $U(I)$ are shown of axially symmetric steady-state modes of current transfer to an axially symmetric tungsten cathode in the form of a rod of radius $R=750 \mu \mathrm{m}$ and a height of $12 \mathrm{~mm}$ (here, $U$ is the near-cathode voltage drop, and $I$ is the arc current). The cathode has a hemispherical tip. A spherical protrusion with a radius of $200 \mu \mathrm{m}$ is located at the top of the tip. The arc operates in argon under the pressure of 2 bar. Also shown is the dependence of the maximum temperature of the cathode surface on the arc current. Stable and unstable sections of each mode are shown by solid or dotted lines, respectively.

There are two separate modes in Fig. 1. One of the modes $(a)$ exists only at low currents and comprises branches separated by

Manuscript received December 10, 2007; revised February 18, 2008. This work was supported in part by FCT, by POCI 2010, and by FEDER under the Projects POCI/FIS/60526/2004 and PPCDT/FIS/60526/2004 Modes of current transfer to cathodes of high-pressure arc discharges and their stability and by FCT, by POCTI-219, and by FEDER under Project Centro de Ciências Matemáticas. The work of M. J. Faria was supported by FCT under Grant SFRH/BD/35883/2007.

The authors are with the Departamento de Física, Universidade da Madeira, 9000 Funchal, Portugal (e-mail: mario@uma.pt).

Color versions of one or more of the figures in this paper are available online at http://ieeexplore.iee.org.

Digital Object Identifier 10.1109/TPS.2008.920888 a turning point. The branch that corresponds to higher values of the near-cathode voltage drop and lower values of the maximum temperature is stable; the branch that corresponds to lower values of the voltage drop and higher temperatures is unstable. The other mode $(b)$ exists in a broad range of currents and is $\mathrm{S}$-shaped at currents between 12 and $21 \mathrm{~A}$. This mode is stable with exception of the section of the S-shape comprised between the turning points.

Apart from this S-shape, the current-voltage characteristics shown in Fig. 1 are similar to those calculated for a cathode in the form of a cylindrical rod with a flat top [4]. On the basis of this similarity, one would expect that the mode $a$ is the axially symmetric spot mode, and the mode $b$ is diffuse. However, high values of the maximum temperature in mode $b$ and low values of the maximum temperature on both branches of the mode $a$ do not fit this interpretation.

An inspection of distributions of the temperature of the cathode, shown for several states in Fig. 1, disproves such interpretation. While high-current states belonging to the mode $b$ are characterized by smooth temperature distributions typical for the diffuse mode, there is a well-defined spot in lowcurrent states belonging to the mode $b$. The stable branch of the mode $a$ is diffuse, and there seems to be something that can be identified as a poorly pronounced spot on the unstable branch, although this spot can hardly be seen in Fig. 1. In other words, each of the two modes of steady-state current transfer existing in this geometry embraces diffuse states and states with spots, which is an unexpected result that has never been reported.

A further investigation allows one to identify a reason of this phenomenon. Calculations performed for variable radius $R$ reveal that a bifurcation of a special type occurs at $R=705 \mu \mathrm{m}$. This bifurcation is similar to the transcritical bifurcation except that the two families of solutions cross at a zero angle (rather than at a finite angle, which is the case for a transcritical bifurcation), i.e., are tangent. There are two disconnected modes at $R<705 \mu \mathrm{m}$, one of these modes being diffuse and the other being the spot mode. At the bifurcation point $R=$ $705 \mu \mathrm{m}$, the modes become connected and exchange branches, and this is why the two modes at $R>705 \mu \mathrm{m}$ (which are disconnected again) embrace states typical for both diffuse and spot modes.

The conclusion that there is only one stable mode in a wide current range, and this is a spot mode, seems to be related with the so-called super spot mode observed in the experiment [2] and, with the experimental observations [3], indicating that a protrusion on the front surface of the cathode facilitates a stable operation on the cathode in the spot mode. 


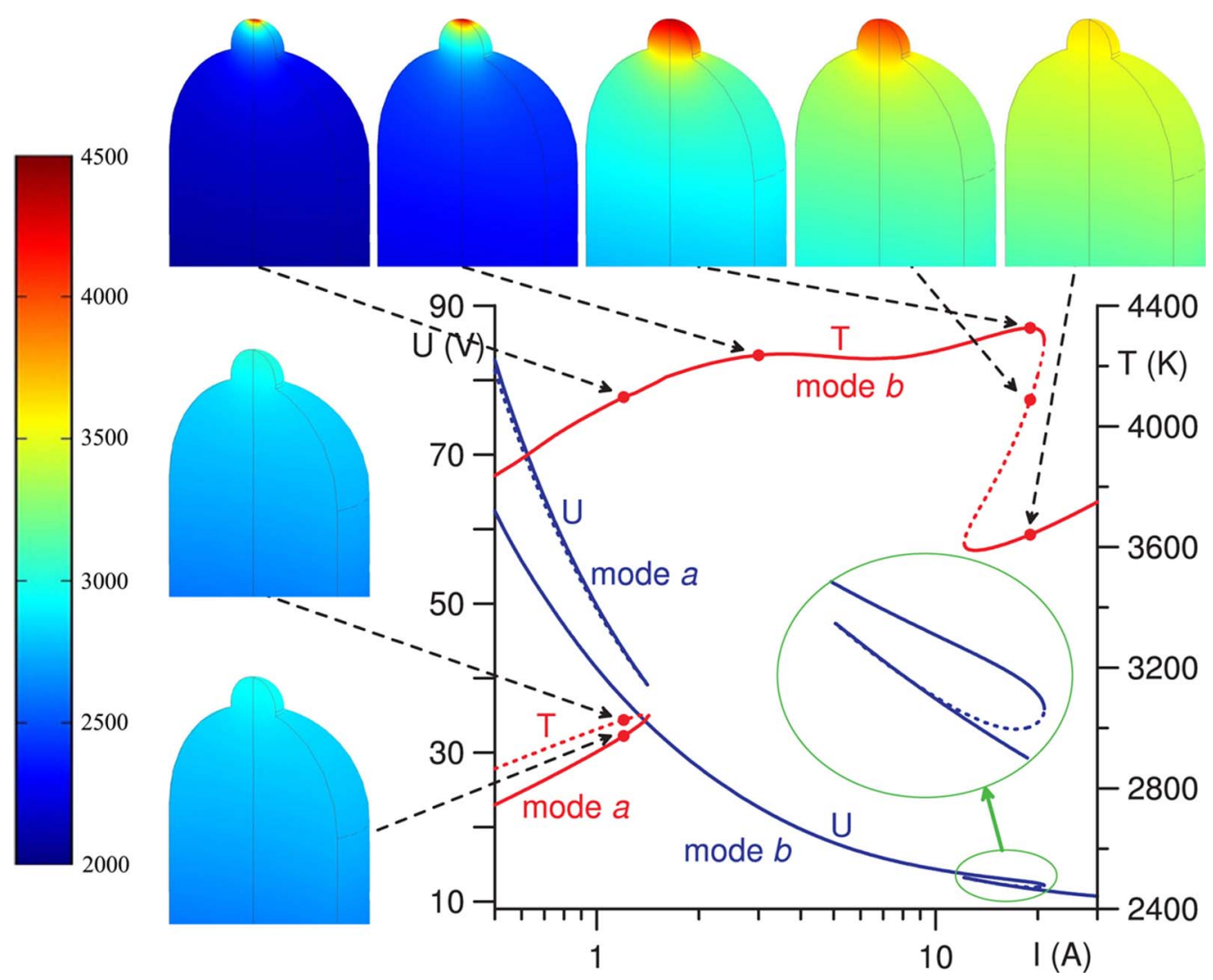

Fig. 1. Current-voltage characteristic and dependence of the maximum temperature of the cathode surface on the arc current for different modes of current transfer and distributions of the temperature of the cathode for several states (the bar in Kelvin).

\section{REFERENCES}

[1] M. S. Benilov, "Understanding and modeling plasma-electrode interaction in high-pressure arc discharges: A review," J. Phys. D, Appl. Phys., vol. 41, no. 14, p. 144001 , Aug. 2008.

[2] T. Hartmann, K. Günther, S. Lichtenberg, D. Nandelstädt, L. Dabringhausen, M. Redwitz, and J. Mentel, "Observation of an extremely constricted cathodic arc attachment to electrodes of high intensity discharge lamps," J. Phys. D, Appl. Phys., vol. 35, no. 14, pp. 1657-1667, Jul. 2002.
[3] L. Dabringhausen, U. Hechtfischer, T. Vos, W. van Erk, M. Haacke, "Electrode behavior in XenEco D4 Hg-free automotive headlight lamps," in Proc. 11th Int. Symp. Sci. Technol. Light Sources (LS), 2007, pp. 529-530.

[4] M. S. Benilov, M. Carpaij, and M. D. Cunha, "3D modelling of heating of thermionic cathodes by high-pressure arc plasmas," J. Phys. D, Appl. Phys., vol. 39, no. 10, pp. 2124-2134, May 2006.

[5] M. S. Benilov and M. J. Faria, "Stability of direct current transfer to thermionic cathodes: II. Numerical simulation," J. Phys. D, Appl. Phys., vol. 40, no. 17, pp. 5083-5097, Aug. 2007. 